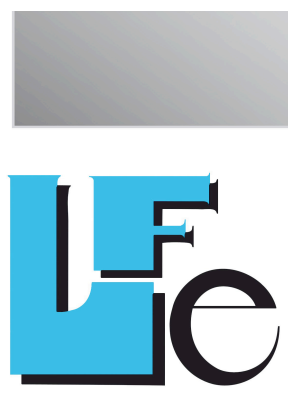

Revista de Lenguas para Fines

Específicos

\section{Revista de lenguas} para fines específicos

elSSN: 2340-8561

Journal information, indexing and abstracting details, archives, and instructions for submissions:

https://ojsspdc.ulpgc.es/ojs/index.php/LFE/index

\section{El desarrollo de la competencia comunicativa intercultural a través del aprendizaje cultural en los libros de texto.}

\section{Antonio R- Raigón-Rodríguez}

Universidad de Córdoba, Facultad de Filosofía y Letras, Plaza del Cardenal Salazar, 3. 18071, Córdoba (Spain).

Article first published online: 27 July 2015.

Article published online with DOI added: 5 April 2016

Revista de Lenguas para fines específicos is licensed under a Creative Commons ReconocimientoNoComercial-SinObraDerivada 4.0 Internacional License. 


\title{
El desarrollo de la competencia comunicativa intercultural a través del aprendizaje cultural en los libros de texto ${ }^{1}$
}

\author{
Antonio R. Raigón-Rodríguez ${ }^{2}$ \\ Universidad de Córdoba
}

\begin{abstract}
Nowadays we live in an intercultural society and this should imply a revision of the language learning approach. Since a good level of linguistic competence does not guarantee successful communication, many scholars have turned to intercultural communicative competence (ICC), a trend reinforced by the Common European Framework of References for Languages (2001). Textbooks are the most important tool for the foreign language teacher (Sercu, 2000). Thus, it should be analyzed whether books are effective for cultural learning as part of ICC development. Following Paige (1999) and Lee (2009), we have designed an itemized three-category model (Culture in general, "big C" culture" and "small c" culture) to analyze three B2-level English textbooks. Using this model, we have recorded a number of incidences per item and per category in each book, differentiating between productive and receptive skills (speaking, writing, listening and reading). At the end of this paper, the results will be discussed regarding the different textbooks, categories and skills, highlighting the most relevant information in each case.
\end{abstract}

Keywords: Intercultural communication, language teaching, textbooks, culture, cultural learning

\section{Resumen}

La omnipresencia de la comunicación intercultural en nuestra sociedad hace necesario un cambio de enfoque en la enseñanza de idiomas. La mera corrección léxico-gramatical no es

$1 \quad$ Title in English: The development of intercultural communicative competence through cultural learning in textbooks.

2 Corresponding author - Universidad de Córdoba, Facultad de Filosofía y Letras, Plaza del Cardenal Salazar, 3. 18071, Córdoba (Spain).

Email: antonio.raigon@uco.es 
suficiente para garantizar una comunicación plena. Con este fin, numerosos expertos han girado hacia la llamada competencia comunicativa intercultural $(\mathrm{CCl})$, hecho ya reflejado a principios de la década pasada en el Marco Común Europeo de Referencia para las Lenguas: Aprendizaje, Enseñanza, Evaluación (MCERL, 2002). El libro de texto supone la guía más importante para el docente en el aula de lengua extranjera (Sercu, 2000). Por lo tanto, debemos analizar si los libros son eficientes para llevar a cabo la enseñanza cultural necesaria para el desarrollo integral de la CCl. Siguiendo a Paige (1999) y Lee (2009), hemos diseñado un modelo basado en ítems divididos en tres categorías (cultura en general, "Cultura" con mayúsculas y "cultura" con minúsculas) y hemos analizado tres libros textos de nivel B2. Mediante este modelo, hemos obtenido el número de incidencias de cada ítem y categoría en los diferentes libros, haciendo diferenciación por destrezas comunicativas (producción oral y escrita, y recepción oral y escrita). Este trabajo acaba con una comparativa de los resultados por libro, categoría y destreza, ofreciendo una evaluación general de los puntos fuertes y las deficiencias encontradas.

Palabras clave: comunicación intercultural, enseñanza de lenguas, libros de texto, cultura, aprendizaje cultural

\section{Introducción}

Desde hace unos años resulta obvio que vivimos en un mundo en el que las relaciones interculturales son cada vez más frecuentes. Muchos especialistas (Sercu, 2005; Byram, 2001; Kramsch, 1998) han insistido en la necesidad de cambiar el enfoque de la enseñanza de idiomas por este motivo. Atrás quedó el tiempo en el que parecía suficiente poder comunicarse con corrección y exactitud gramatical. Además, el objetivo ideal de la reproducción del acento nativo plantea muchos interrogantes, que son más en el caso del inglés: ¿Acento nativo de dónde? ¿Merece la pena el esfuerzo? ¿Es suficiente tener un dominio perfecto sin conocimientos culturales?

La sociedad actual requiere que los alumnos sean interculturalmente competentes y que puedan relacionarse de manera completa con personas con diferentes bagajes socioculturales. Para evitar conflictos interculturales, es necesario ser consciente de los valores y creencias del otro interlocutor, venga de donde venga. Entendemos, pues, que las enseñanzas lingüística y cultural han de ir de la mano. Así, nuestro estudio comenzará definiendo el concepto de cultura.

Seguidamente, revisaremos la evolución del enfoque comunicativo para la enseñanza de las lenguas y analizaremos los elementos que conforman la competencia comunicativa intercultural (CCl). En este punto, se hará necesario reseñar las ideas planteadas en el Marco Común Europeo de Referencia para las Lenguas: Aprendizaje, Enseñanza, Evaluación (MCERL, 2002). 
Una vez aclarados estos conceptos, nuestro estudio plantea un análisis del contenido cultural de tres libros de texto partiendo de los modelos presentados por Paige et al. (1999) y Lee (2009). Esto nos servirá para saber qué tipo de enseñanza cultural se está promoviendo dentro del aula de inglés como lengua extranjera.

\section{La cultura y la enseñanza de lenguas}

Nuestro estudio parte de dos consideraciones fundamentales: primero, la consideración conjunta del binomio lengua y cultura, y segundo, la naturaleza visible e invisible de la propia cultura. Para entender los comportamientos y las manifestaciones culturales es necesario conocer los valores y creencias que subyacen bajo estos.

El vínculo existente entre la lengua y la cultura ha sido defendido por numerosos autores (Zarate, 1986; Byram, 1997; Kramsch, 1998). Como afirmaban los estructuralistas norteamericanos de la primera mitad del siglo veinte (Sapir y Whorf, 1958), la lengua que hablamos determina nuestra manera de ver el mundo y nuestra organización mental. De igual manera, la forma en la que somos socializados determina de manera definitiva cómo vemos el resto del mundo que nos es ajeno.

El eminente lingüista norteamericano G. Lakoff (1987) explica cómo el uso metafórico de la lengua hace que los hablantes conceptualicen, de una manera u otra, conceptos que no siempre coinciden en otras culturas y que serían difícilmente traducibles.

Novinger (2001, p.47) ilustra un ejemplo bastante más radical que los arriba expuestos: los Sioux carecen de palabras para expresar "llegar tarde" o "esperar a alguien". Esto, sin duda, refleja y afecta el tratamiento que esta cultura da al tiempo.

En línea con lo expuesto, y en relación al aprendizaje de un idioma extranjero, creemos firmemente que la enseñanza de la lengua y de la cultura se realiza de manera simultánea e interdependiente (Mitchell and Myles, 2005) y no puede separarse (Byram, 1989; Kramsch, 1993). Curiosamente explicativa resulta la analogía de Bennett, Bennett y Allen (2003, p.237) cuando hablan del riesgo de convertirse en un imbécil con fluidez ("becoming a fluent fool" sic) si se aprende la lengua sin conocer su cultura. 
En segundo lugar y en referencia a la naturaleza del concepto mismo, nos parece muy ilustrativa la propuesta de Weaver (1986): la cultura es como un iceberg, los comportamientos externos son una pequeña parte de la realidad completa que supone el hecho cultural, pero son los únicos visibles. Además, esta parte "sumergida", que está compuesta por las creencias y los valores, difícilmente cambia con el paso del tiempo. El análisis de esa parte subyacente, esa visión del mundo particular, nos ayuda a comprender los comportamientos externos. Partiendo de la célebre metáfora del iceberg cultural de Weaver, Pusch y La Brack (2003) argumentan que todo lo que es perceptible y que se sitúa, por así decirlo, por encima del nivel del agua en el caso del iceberg, está compuesto por la "Cultura" con mayúscula. La "cultura" con minúscula estaría compuesta, al contrario, por lo subjetivo, lo complejo, lo no explícito. Esta la componen los valores que se encuentran en la parte oculta del iceberg y que motivan los comportamientos. En su naturaleza menos explícita radica el problema, pues son los más importantes pero lo más difíciles de aprender.

Por otra parte, encontramos la dicotomía presentada por Holliday, Hyde y Kullman (2004) entre la concepción esencialista y no esencialista de la cultura.

Según expone Areizaga (2002) existen dos modelos de enseñanza cultural una vez hemos aclarado el objeto de estudio, una visión informativa y otra formativa. Si el fin último es la formación integral del alumnado, es necesario que optemos por la segunda. Se trata de un modelo constructivista, no exclusivamente factual y no esencialista que busca el desarrollo de las actitudes y las habilidades necesarias para una comunicación plena. Este desarrollo competencial del alumnado se puede producir en el aula de lengua extranjera, pero va más allá de ella, ya que se trata de un aprendizaje personal extrapolable a todos los ámbitos y que permita al alumnado conocer lo que se oculta tras los comportamientos culturales.

\section{El enfoque intercultural en la enseñanza de lenguas}

La diversidad cultural traída con la globalización hace que la competencia comunicativa quede corta en nuestro afán de interactuar con personas de diferentes procedencias (Larzen-Ostermark, 2009). Como acierta en señalar Sercu (2005), toda la comunicación en lengua extranjera es, por definición, intercultural. Una comprensión completa requiere más que una mera transmisión y recepción de signos lingüísticos, ya que la realidad aparece filtrada, tal y como hemos visto, por nuestros valores y creencias culturales. 
El desarrollo de la competencia comunicativa intercultural (CCI) se hace esencial para nuestro alumnado y para su futuro desarrollo como ciudadano y profesional. La enseñanza de idiomas desde el enfoque intercultural enfatiza la importancia de desarrollar la consciencia cultural del alumno con el fin de permitirle comunicarse plenamente con personas culturalmente diversas y ser conocedor de su propio "filtro" cultural.

De la misma manera, es necesario tener en cuenta cuál es realmente nuestra meta. Durante mucho tiempo, la enseñanza de lenguas extranjeras ha estado persiguiendo la innecesaria quimera de la reproducción exacta de hablantes que fuesen reproducciones "clónicas" del hablante nativo. No usamos la expresión "innecesaria quimera" al azar: en condiciones normales, no es posible, aunque algunas personas se acerquen, llegar a dominar un idioma al nivel que dominamos nuestra lengua madre. Además, este enorme esfuerzo rara vez se ve recompensado. Es suficiente comunicarnos de manera autónoma y ajustada a nuestras necesidades. Por otro lado, si nos centramos en el caso del inglés como el idioma que más se aprende como segunda lengua en el mundo, hay muchas posibilidades de que cuando lo usemos para comunicarnos sea con personas cuya primera lengua tampoco sea el inglés. Es decir, imitar de manera exacta uno u otro acento no es garantía de comprensión ni, en la práctica, necesario. Los materiales que sólo muestren hablantes nativos no deben ser exclusivos, ya que la realidad contradice esta premisa (Widdowson, 1998; Liaw, 2006; Alptekin, 2002). Vez (2001, p.8) afirma que la enseñanza de lenguas debe dar una respuesta apropiada a la necesidad de tolerancia y de valores interculturales en nuestra sociedad postmoderna.

El lugar del inglés como lengua de comunicación intercultural (Word Englishes) hace que el objetivo de la enseñanza del idioma no sea ya la imitación del hablante nativo británico o americano.

El nuevo hablante intercultural es una persona que se encuentra cómoda entre personas que tienen bagajes culturales diferentes al suyo, que es capaz de aceptar la diferencia y que no se siente amenazada por ella. Además, es capaz de objetivar sus propias creencias, de negociar, de hacerse entender y hacer un esfuerzo por entender a los demás.

La CCl se define como la habilidad para negociar significados culturales y ejecutar conductas comunicativas eficaces (Rodrigo, 1999). Esta comunicación intercultural se da entre dos o más personas con referentes culturales diferentes que superan alguna barrera contextual o personal para llegar a una comprensión mutua suficiente (Vilà, 2004). Se divide en dos 
dimensiones: la dimensión verbal, que incluye el idioma pero no de manera exclusiva, y la dimensión no verbal, donde podríamos incluir factores como la distancia física o proxémica, el lenguaje corporal o gestual y el silencio, entre otros, que cambian de significado en diferentes culturas. Asimismo, no se describe en términos de perfección, sino de suficiencia.

Aunque muchas propuestas han sido presentadas, el modelo de $\mathrm{CCl}$ que mayor consenso ha obtenido entre los especialistas ha sido la propuesta por Byram (1997; 2001). Tradicionalmente, los acercamientos a la cultura de la L2 en el aula se han centrado en la transmisión de información. Dentro de este enfoque, el aprendizaje va más allá de la mera adquisición factual, teniendo en cuenta el conocimiento, habilidades, actitudes y consciencia cultural.

Byram habla de que los componentes básicos de la CCl son los contenidos conceptuales (savoirs), las distintas habilidades necesarias (savoir comprendre, apprendre/faire, s'engager) y, sobre todo, las actitudes interculturales (savoir être). Son estas últimas las que nos permiten sentir curiosidad, ser abiertos $y$, como él mismo señala, descentralizar (decentre), a través del cuestionamiento de los valores propios. Byram define así la capacidad de descentralizar:"...a willingness to suspend belief in one's own meanings and behaviours, and to analyse them from the viewpoint of the others with whom one is engaging."(1997, p.34)

De esta manera, hemos de adquirir una perspectiva más objetiva de nuestros propios valores, que damos por sentados la mayoría de las veces. Según este autor, la habilidad de descentralizar y la actitud positiva son las columnas que sostienen la comunicación intercultural, extremo que apoya Deardroff (2006).

\begin{tabular}{l|l}
\hline $\begin{array}{l}\text { Contenidos } \\
\text { conceptuales }\end{array}$ & $\begin{array}{l}\text { No se trata de conocer información de una cultura particular, sino } \\
\text { más bien de conocer cómo funcionan los grupos sociales. }\end{array}$ \\
\hline Actitudes & $\begin{array}{l}\text { Éstas incluyen la curiosidad, la tolerancia y el respeto a la alteridad. } \\
\text { Es necesario estar dispuesto a cuestionar los valores propios, } \\
\text { olvidando las tendencias naturales etnocentristas. }\end{array}$ \\
\hline $\begin{array}{l}\text { Habilidades de } \\
\text { descubrimiento e } \\
\text { interacción }\end{array}$ & $\begin{array}{l}\text { Nos hacen capaces de adquirir conocimiento cultural nuevo y } \\
\text { llevarlo a cabo durante el transcurso de una interacción } \\
\text { intercultural y bajo la presión de la misma. }\end{array}$ \\
\hline $\begin{array}{l}\text { Habilidades de } \\
\text { interpretación y } \\
\text { relación }\end{array}$ & $\begin{array}{l}\text { Nos permiten interpretar un evento o un documento de otra } \\
\text { cultura, para explicarlo y relacionarlo con documentos o eventos } \\
\text { de la nuestra. }\end{array}$ \\
\hline $\begin{array}{l}\text { Conciencia cultural } \\
\text { crítica }\end{array}$ & $\begin{array}{l}\text { Habilidad para evaluar críticamente prácticas y productos de otras } \\
\text { culturas y de la nuestra propia, basándose en criterios objetivos. }\end{array}$ \\
\hline
\end{tabular}

Tabla 1. Componentes de la CCI (Byram, 1997; 2001). 
En un proceso continuo de aprendizaje, los alumnos pasan por un análisis crítico de sus propios valores, una aceptación de los ajenos hasta un momento de apreciación de la diferencia alejándose del etnocentrismo (Byram, 2000).

\section{Los libros de texto y el aprendizaje cultural}

Aunque se puedan encontrar detractores, el libro de texto sigue constituyendo el eje vertebrador de la enseñanza de idiomas dentro del aula (Sercu, 2000). Este proporciona al docente una guía que puede ser una ayuda $y$, al mismo tiempo, un riesgo, que supone la excesiva mecanización de la labor del docente y la menor respuesta a las necesidades singulares de nuestros alumnos. Uno de los problemas que nos presentan los libros de texto es una visión fragmentada y estereotipada de la cultura extranjera (Paricio, 1996), una mera transmisión de información objetiva que queda lejos de reflejar la realidad de un concepto tan complejo. A menudo, suponen la superficial visión de un "turista". Además, este tipo de enseñanza cultural se basa en una ecuación errónea que iguala la enseñanza cultural a la enseñanza de la cultura de la L2, asociada a un grupo determinado de personas (Risager, 1998).

Efectivamente, este enfoque de enseñanza monocultural es intranscendente e inútil para el fin de la enseñanza intercultural que debe suponer el objetivo principal, ya que niega la complejidad y la mutabilidad de la cultura.

El desarrollo de la competencia comunicativa intercultural es donde encontramos la respuesta a la multiplicidad de culturas que podemos encontrar en un aula de lengua extranjera: la propia, la angloparlante o la del hablante de otra cultura que usa el inglés como lengua internacional al igual que nosotros (Hyde, 1998; Widdowson, 1998; Alptekin, 2002).

Es por ello que el quid de la cuestión no es saber la "cultura de quién" hay que enseñar sino el desarrollo de la CCI (Byram, 1997). Para ello, el primer paso es el conocimiento de la cultura propia. Sin autoconsciencia de esta no puede haber enseñanza cultural.

De igual manera, no es tan importante que nuestros alumnos aprendan una u otra cultura, sino que se familiaricen con conceptos generales y se conviertan en hablantes culturalmente "competentes" (Lee, 2012, p.193). 
Según Varón (2009, p.100), las grandes editoriales en sus libros de texto han dedicado todo su esfuerzo a desarrollar las denominadas cuatro destrezas del alumnado, dedicando publicaciones especiales a la cultura, como si se tratase de un ente separado de la enseñanza de idiomas. Según la propia autora, la mayoría de las situaciones culturales presentadas no incitan el debate, al tratarse de problemas que se presentan completamente solucionados.

Por otra parte, Kramsch (1993) recuerda que este aspecto cultural ha sido marginado en pos de lo estructural o funcional. Esta autora señala que esto se debe a la histórica "tiranía" de la lingüística en el campo de la didáctica de las lenguas, entorpeciendo la adopción de un enfoque más holístico. Vez (2001) abunda en esta aseveración y en la influencia de la lingüística en el diseño de los libros de texto. De este modo, las diferentes corrientes se han visto reflejadas en los libros de las distintas épocas.

No debemos olvidar la influencia reciente de la publicación del MCERL (2002) en la creación de materiales curriculares para el aula de lenguas. Su publicación supuso una estructuración y un acuerdo generalizado sobre los niveles que han de lograrse para alcanzar una determinada competencia comunicativa.

Los autores del mismo reconocen la importancia del hecho intercultural, y lo incluyen dentro de los saberes necesarios de la competencia comunicativa, incluyéndolo como "consciencia intercultural": "El conocimiento, la percepción y la comprensión de la relación entre el mundo de origen y el mundo de la comunidad objeto de estudio (similitudes y diferencias distintivas) producen una consciencia intercultural" (2001, p.101).

A las consabidas competencias comunicativas, el MCERL (2002, p.98-124) añadió la sociolingüística y la pragmática.

\begin{tabular}{|c|c|}
\hline \multirow{5}{*}{$\begin{array}{l}\text { Competencia } \\
\text { Sociolingüística }\end{array}$} & Los marcadores lingüísticos de relaciones sociales. \\
\hline & Las normas de cortesía. \\
\hline & Capacidad de entender las expresiones de sabiduría popular. \\
\hline & Capacidad de reconocer las diferencias de registro. \\
\hline & Capacidad de reconocer las diferencias de acento y dialecto. \\
\hline \multirow{2}{*}{$\begin{array}{l}\text { Competencia } \\
\text { Pragmática }\end{array}$} & $\begin{array}{l}\text { Competencia discursiva o capacidad de ordenar oraciones para } \\
\text { producir fragmentos coherentes de lengua. }\end{array}$ \\
\hline & $\begin{array}{l}\text { Competencia funcional o capacidad de establecer una } \\
\text { interacción coherente dentro de intercambios comunicativos } \\
\text { sociales. }\end{array}$ \\
\hline
\end{tabular}

Tabla 2. Competencias no lingüísticas. 
Sin embargo, su definición de la competencia sociolingüística queda reducida al tratamiento lingüístico de dichos populares expresiones coloquiales y diferentes registros y variedades sociales (Göbel y Helmke, 2010). Esto queda lejos de la concepción completa del hablante intercultural: una persona que conoce, acepta y aprecia las diferencias culturales entre los participantes en un acto comunicativo.

Un análisis profundo del tratamiento de la cultura en los textos nos parece un primer paso esencial a la hora de plantearnos qué tipo de praxis estamos llevando a cabo en el aula de lengua extranjera. La enseñanza de la lengua en desconexión de su cultura es una enseñanza incompleta (Genc and Bada, 2005). El objetivo de este estudio es, pues, realizar ese análisis con el fin de determinar qué tipo de enseñanza cultural se está llevando a cabo a través de los materiales curriculares y con esto saber si se está completando la competencia comunicativa del alumnado en los términos explicitados por el MCERL (2002).

\section{Método}

Hemos seleccionado tres libros de nivel B2 para adultos universitarios de las tres editoriales utilizadas con más frecuencia en el mercado nacional español en la enseñanza de inglés como lengua extranjera: Cambridge University Press, Macmillan y Oxford University Press. Los textos son English Unlimited (2011), Straight Forward (2012) y New English File (2008), respectivamente. Los libros corresponden a la edición más reciente disponible en el momento. La nueva edición de English File no está aún disponible en su nivel upperintermediate (B2).

La elección del nivel B2 se debe a que la mayoría de los alumnos universitarios que acceden a becas de movilidad (Erasmus, Leonardo...) necesitan acreditar este nivel o superior para ser seleccionados.

Para analizar los elementos culturales e interculturales en los libros de texto escogidos, hemos partido del modelo propuesto por Paige et al (1999) y Lee (2009). El modelo conceptual de aprendizaje cultural de Paige (1999, p.54) presenta una distinción entre el aprendizaje de una cultura específica (culture-specific) y el de la cultura general (culture-general), tratándose este último de aquellas destrezas y conocimiento de naturaleza más generalizable y transferible entre culturas. Este aprendizaje es esencial para capacitar al alumno a aprender en cualquier contexto que resulte culturalmente diferente (Byram, 1997; Kramsch, 2005). También presenta una división entre 
conocimientos, comportamientos y actitudes, heredada de los conceptos defendidos por autores como Kramsch y Byram, que se encuentran en consonancia con nuestra idea de desarrollo de la CCl.

Lee (2009) introduce en su análisis dos distinciones más: "Cultura" con mayúscula y "cultura" con minúscula (Pusch y La Brack, 2003), ya tratada anteriormente en este trabajo, y una división en temas que hace más objetivable y aplicable el modelo al análisis de materiales curriculares. Además, realiza una división de tipo formal: diálogo, imagen o actividad, dependiendo de cómo se presenta el aspecto cultural en el libro.

Aunque supone un buen punto de partida, nos parece que Lee (2009) plantea un modelo demasiado exhaustivo para ser práctico a la hora de analizar los materiales. Él propone la búsqueda de 64 temas diferentes, en cualquier forma dentro de los libros. Hemos simplificado el número de temas y hemos considerado oportuno dividir los resultados por destrezas, siguiendo la división formal que se adopta en la mayoría de libros de texto para la enseñanza de idiomas.

En este trabajo, se lleva a cabo un análisis cuantitativo basado en la observación y anotación de incidencias culturales. Los datos reflejados en las tablas se han obtenido consignando el número de incidencias culturales en los diferentes manuales. Así, cada tabla presenta los diferentes temas propuestos en el modelo para el aprendizaje cultural divididos por destrezas. En cada casilla figura el número total de incidencias por tema. En caso de que en una misma actividad se trabajen diferentes temas culturales, se ha anotado de manera independiente.

A continuación, pasaremos a analizar los resultados obtenidos dentro de las tres dimensiones que conforman este modelo: cultura en general, categorías pertenecientes a la "Cultura" con mayúsculas y categorías pertenecientes a la "cultura" con minúsculas.

\section{Análisis de resultados}

\subsection{Categorías pertenecientes a la "Cultura en general"}

El aprendizaje cultural general se encuentra dividido en tres dimensiones: el conocimiento, los comportamientos y las actitudes. Es la información cultural, el conocimiento propiamente dicho, el que ocupa un lugar prioritario con 100 incidencias totales en los tres libros, seguido muy de lejos 
por las referencias a las actitudes y los comportamientos, apenas tratados (con 21 y 19 incidencias respectivamente). Individualmente, es el aprendizaje cultural de hechos objetivos el que tiene mayor protagonismo (55 veces encontrado en los textos). También han de señalarse las ocasiones en las que se trabaja el desarrollo intercultural, la identidad cultural propia y el desarrollo de la actitud positiva hacia el aprendizaje cultural (20, 21 y 13, respectivamente). Esto supone continuar con una tendencia ya detectada en pasadas décadas por los expertos.

\begin{tabular}{|c|c|c|c|c|c|c|c|c|c|c|c|c|c|}
\hline \multirow{2}{*}{\multicolumn{2}{|c|}{ Cultura en general }} & \multicolumn{4}{|c|}{$\begin{array}{l}\text { NEW ENGLISH } \\
\text { FILE } \\
\text { UPPER-INT. }\end{array}$} & \multicolumn{4}{|c|}{$\begin{array}{l}\text { ENGLISH } \\
\text { UNLIMITED } \\
\text { UPPER-INT. }\end{array}$} & \multicolumn{4}{|c|}{$\begin{array}{l}\text { STRAIGHTFORWARD } \\
\text { UPPER-INT. }\end{array}$} \\
\hline & & $R$ & $L$ & $S$ & $w$ & $R$ & $L$ & $S$ & $W$ & $R$ & $L$ & $S$ & $w$ \\
\hline \multirow{4}{*}{$\begin{array}{l}\text { O } \\
\sum \\
\text { 岂 } \\
\sum \\
\text { U } \\
\text { ○ } \\
\text { O }\end{array}$} & Aprendizaje cultural & 10 & 2 & & & 7 & 11 & 9 & 1 & 10 & 4 & 1 & \\
\hline & Fases del ajuste cultural (Shock) & 1 & & & & 1 & 1 & 1 & & & & & \\
\hline & Desarrollo intercultural & & & & & 3 & 3 & 5 & & 2 & & 7 & \\
\hline & Identidad cultural & & & 6 & & & & 13 & 2 & & & & \\
\hline \multirow{4}{*}{ 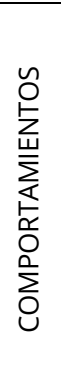 } & $\begin{array}{l}\text { Estrategias de aprendizaje } \\
\text { cultural }\end{array}$ & & & & & 1 & 1 & 4 & & & & & \\
\hline & $\begin{array}{l}\text { Estrategias para mediar con el } \\
\text { estrés intercultural }\end{array}$ & & & & & 1 & & 1 & & & & & \\
\hline & $\begin{array}{l}\text { Competencia comunicativa } \\
\text { intercultural }\end{array}$ & & & & & 1 & 2 & 4 & 1 & & & & \\
\hline & Adaptabilidad cultural & & & & & 1 & & 2 & & & & & \\
\hline \multirow{2}{*}{ 岂 } & $\begin{array}{l}\text { Actitud positiva hacia el } \\
\text { aprendizaje cultural }\end{array}$ & 1 & & 1 & & 1 & 2 & 6 & & & 1 & 1 & \\
\hline & $\begin{array}{l}\text { Actitud positiva hacia las } \\
\text { diferencias culturales }\end{array}$ & 1 & & & & 1 & 1 & 5 & & & & & \\
\hline
\end{tabular}

Tabla 3. Incidencias en el apartado de "Cultura en general".

Sin embargo, merece la pena mencionar las diferencias entre los tres libros en este sentido. Para comenzar, de las 140 incidencias referenciadas entre Ios tres libros, English Unlimited (EU) presenta 92, lo que supone un 65,7\% del total, frente al 15,7\% de New English File (NEF) y 18,6\% de Straight Forward (SF). Las diferencias se hacen aún más agudas cuando contabilizamos sólo los comportamientos y actitudes, que desde nuestro punto de vista son las dimensiones que han de ser más atendidas en el 
desarrollo de la CCI: El 100\% de las incidencias referidas al comportamiento y el $76,2 \%$ de las actitudes corresponden a EU.

Si atendemos a las diferencias por destrezas, hemos de señalar que NEF y SF usan mayoritariamente las lecturas para introducir los temas de cultura general, mientras que EU los trabaja en más de la mitad de las ocasiones en ejercicios de producción oral. En general, las destrezas productivas (lectura y audiciones) se usan en un $50 \%$ de las incidencias totales. Sin embargo, la destreza más utilizada es la productiva oral, lo que nos hace deducir que esta dimensión de aprendizaje cultural ofrece enorme potencialidad en las discusiones y role-plays dentro del aula. La producción escrita se usa de manera bastante marginal (2,8\% del total) con este fin. Este dato muestra una carencia importante debido a que se evita que los alumnos dediquen un mayor tiempo de reflexión a los temas presentados. Además, las incidencias en esta destreza están relacionadas con el aprendizaje de contenidos casi exclusivamente.

\subsection{Categorías pertenecientes a la “Cultura” con mayúsculas}

Como se podía avanzar antes de realizar el estudio, la dimensión más superficial de la cultura específica, la "Cultura" con mayúsculas, aparece con mayor frecuencia en los tres textos. Las quince categorías son tratadas de manera desigual, siendo las más referenciadas en general las obras literarias, cinematográficas, etc., las costumbres sociales, los grupos étnicos, los negocios y las transacciones económicas, la artesanía y la arquitectura, la geografía, las ciencias y la vida urbana. Todas estas conforman una realidad fácilmente reconocible a simple vista. Es más, el enfoque carece de profundidad y ofrece únicamente un conocimiento anecdótico. Sorprendentemente poco tratadas son la familia, la comida, el vestir y la comunicación no verbal. Se trata de aspectos esenciales en la comunicación intercultural en nuestra opinión, mucho más que una canción en particular, un monumento o la geografía de un grupo humano determinado. Son precisamente en los aspectos no verbales donde encontramos la mayor parte de los malentendidos entre personas con referentes culturales distintos.

Aquí, también, encontramos grandes diferencias entre los textos. De las 203 incidencias totales registradas, más de la mitad corresponden a EU (106), seguido por SF (51) y NEF (46). Es más, el primero trata todas las dimensiones que hemos decidido analizar. 


\begin{tabular}{|c|c|c|c|c|c|c|c|c|c|c|c|c|}
\hline \multirow[t]{2}{*}{$\begin{array}{l}\text { Categorías pertenecientes a la "Cultura" con } \\
\text { mayúsculas }\end{array}$} & \multicolumn{4}{|c|}{$\begin{array}{l}\text { NEW } \\
\text { ENGLISH FILE } \\
\text { UPPER-INT. }\end{array}$} & \multicolumn{4}{|c|}{$\begin{array}{l}\text { ENGLISH } \\
\text { UNLIMITED } \\
\text { UPPER-INT. }\end{array}$} & \multicolumn{4}{|c|}{$\begin{array}{l}\text { STRAIGHTFORWARD } \\
\text { UPPER-INT. }\end{array}$} \\
\hline & $R$ & $L$ & $S$ & $W$ & $R$ & $L$ & $S$ & $W$ & $\boldsymbol{R}$ & $L$ & $S$ & $w$ \\
\hline Grupos étnicos & 2 & 1 & & & 5 & 9 & & & 2 & & & \\
\hline Geografía/historia/política/variaciones regionales & 1 & & & & 2 & 3 & 1 & & 5 & 3 & & \\
\hline Arte/artesanía/monumentos y lugares históricos & & 2 & & & 4 & 3 & 2 & 1 & 2 & 1 & 1 & \\
\hline Literatura/cine/música/medios de comunicación & 3 & 9 & 3 & & 5 & 2 & 1 & 1 & 5 & 2 & 3 & \\
\hline Medicina/Ciencia & 2 & 1 & 1 & & 3 & 3 & 2 & & 3 & & & \\
\hline Moneda/compras/Mercado/industria/negocios & 2 & & & & 5 & 4 & 1 & 1 & 2 & 1 & 1 & \\
\hline $\begin{array}{l}\text { Vida } \\
\text { urbana/infraestructuras/alojamientos/transporte }\end{array}$ & 2 & 4 & & 1 & 2 & 3 & & & 2 & 1 & & \\
\hline Educación & & & & & 3 & 3 & 2 & & & & & \\
\hline Vestimenta & 1 & & 1 & & 2 & & & & 2 & & & \\
\hline Comida & & & & & 2 & 2 & & & & & & \\
\hline Festividades/ fiestas/celebraciones/ceremonias & 1 & & & & 2 & 3 & 2 & & 1 & & & \\
\hline Costumbres sociales & 3 & 2 & 2 & 1 & 2 & 8 & 3 & & 4 & & 2 & \\
\hline Tiempo libre/deportes & & & & & 1 & 1 & & & 2 & 2 & 2 & \\
\hline Familia & & & & & & 3 & & & 1 & & & \\
\hline $\begin{array}{l}\text { Comunicación no verbal (espacio personal, } \\
\text { oculésica, háptica...) }\end{array}$ & 1 & & & & 2 & 2 & & & 1 & & & \\
\hline
\end{tabular}

Tabla 4. Incidencias en el apartado de "Cultura" con mayúsculas.

Analizando las cifras generales por destrezas, estas categorías aparecen principalmente en las destrezas receptivas (lectura $44 \%$ y audición 38\%), quedando las productivas relegadas a un $15 \%$ en el caso de la oral y un escaso $2 \%$ en escrita. Esto nos hace concluir que el procedimiento que se lleva a cabo es la transmisión de conocimientos, más que la interrelación o concienciación de los alumnos en la cultura de la segunda lengua y la propia. El poco uso de las destrezas productivas hace que los alumnos no estén involucrados completamente en el proceso de aprendizaje ni puedan incorporar sus propios conocimientos culturales a dicho proceso. La reflexión y discusión sobre lo aprendido no llega a darse en la mayoría de los casos, ya que no existen actividades de producción por parte del alumnado.

\subsection{Categorías pertenecientes a la "cultura" con minúsculas}

Dentro del estudio de una cultura específica, la "cultura" con minúsculas ha de ser el fin último de los estudiantes, en tanto que esta contiene los valores 
y las creencias que subyacen bajo los comportamientos externos que vemos en los artefactos o manifestaciones culturales.

Estas categorías, según nuestro estudio, se encuentran bastante descuidadas en los libros que hemos seleccionado. Se trata de un aprendizaje mucho más complicado y al que hay que dedicar un mayor esfuerzo dada su naturaleza menos observable.

\begin{tabular}{|c|c|c|c|c|c|c|c|c|c|c|c|c|}
\hline \multirow[t]{2}{*}{$\begin{array}{l}\text { Categorías pertenecientes } \\
\text { a la "cultura" con } \\
\text { minúsculas }\end{array}$} & \multicolumn{4}{|c|}{$\begin{array}{l}\text { NEW ENGLISH } \\
\text { FILE } \\
\text { UPPER-INT. }\end{array}$} & \multicolumn{4}{|c|}{$\begin{array}{l}\text { ENGLISH } \\
\text { UNLIMITED } \\
\text { UPPER-INT. }\end{array}$} & \multicolumn{4}{|c|}{$\begin{array}{l}\text { STRAIGHTFORWARD } \\
\text { UPPER-INT. }\end{array}$} \\
\hline & $R$ & $L$ & $S$ & $w$ & $R$ & $L$ & $S$ & $w$ & $\boldsymbol{R}$ & $L$ & $S$ & $w$ \\
\hline Individualismo/colectivismo & & 1 & & & & 3 & 1 & & 1 & & & \\
\hline Igualdad & & & & & 1 & 3 & 2 & & 1 & 1 & & \\
\hline Justicia & 1 & 1 & 1 & & 1 & 2 & 3 & & 2 & & 1 & \\
\hline Competitividad & & & & & 1 & 2 & & & 1 & & & \\
\hline Materialismo & 1 & 1 & & & & 1 & 1 & & 1 & & 1 & \\
\hline Confrontación & & & & & 1 & 2 & 2 & & & & 1 & \\
\hline Orientación hacia lo nuevo & & & & & & 2 & & & & & & \\
\hline Auto-mejora & & 1 & & & 3 & & & & 2 & & & \\
\hline Crianza & 1 & & & & 2 & 5 & 2 & & 1 & & & \\
\hline Tiempo & 1 & & & & & & & & & & & \\
\hline Nivel de formalismo & & & & & 2 & 4 & 2 & & 1 & & & \\
\hline $\begin{array}{l}\text { Estilos comunicativos: } \\
\text { directo-indirecto }\end{array}$ & & & & & 2 & 4 & 2 & & & & & \\
\hline $\begin{array}{l}\text { Grado de orientación hacia } \\
\text { las normas }\end{array}$ & 1 & 1 & 1 & & 1 & 2 & 2 & & & & & \\
\hline Masculinidad & & & & & & & & & 1 & 1 & & \\
\hline $\begin{array}{l}\text { Grado de orientación hacia } \\
\text { los resultados }\end{array}$ & & & & & & 3 & & & 2 & & & \\
\hline Importancia del trabajo & & & & & 2 & & & & & & & \\
\hline
\end{tabular}

Tabla 5. Incidencias en el apartado de "cultura" con minúsculas.

Aunque el tratamiento en los tres libros es muy diferente, hemos de detenernos a analizar los datos generales. En comparación con las anteriores dos dimensiones, el número de incidencias es relativamente pequeño (96, frente a 140 y 203). Sólo se han encontrado más de 10 incidencias en los apartados concepción de justicia y la de crianza. Las demás son tratadas de puntillas cuando no ignoradas.

Es más, si dejásemos aparte EU, el número de incidencias en los otros dos textos es absolutamente testimonial: 12 en NEF y 18 en SF. Aquí, de nuevo, EU se destaca bastante en su tratamiento del aprendizaje cultural. Esta es 
una consecuencia lógica de lo anteriormente señalado. Además de las categorías mencionadas, se hace hincapié en la igualdad, la confrontación, el nivel de formalidad, los estilos comunicativos y el nivel de importancia de las normas, todas con más de 5 incidencias registradas. Sólo quedan el tiempo y el nivel de masculinidad sin tratar de las categorías analizadas en este estudio. Con un número de incidencias totales de 66, EU casi cuadriplica a SF y casi sextuplica a NEF.

Teniendo en cuenta la distribución por destrezas comunicativas, las receptivas vuelven a ser protagonistas con un $77 \%$ de las incidencias $(74,2 \%$ en EU), dejando el restante $25 \%$ para la producción oral. La producción escrita no aparece registrada en ningún caso. De nuevo, observamos una tendencia unidireccional en la enseñanza de contenidos culturales. Queda poco espacio para el debate y la reflexión de este tipo de contenidos dentro del currículo presentado en los textos. El efecto de esta tendencia ha de ser forzosamente el mismo. La construcción de un aprendizaje significativo en el aula resulta más complicada cuando lo único que se hace es un aplicar un proceso de mera transmisión de información.

\section{Discusión de resultados}

En NEF, los ejemplos referidos a la "Cultura" con mayúsculas aparecen deslavazados, como es el caso de las canciones que se presentan al final de las unidades. Cuando se tratan temas que pueden resultar interesantes por la cantidad de aprendizaje y debate que pueden suscitar (por ejemplo, Amish in the city, p.88), el tratamiento del mismo queda en lo superficial. En la actividad a la que hacemos referencia, se habla de un grupo de jóvenes de una comunidad Amish que empieza a vivir en la ciudad de Los Ángeles, California. En lugar de usar este ejemplo de choque cultural para propiciar el debate, la actividad acaba con preguntas de comprensión lectora referidas al texto, quedando en lo meramente anecdótico sin aprovechar la potencialidad de la situación.

Macmillan presenta con SF un libro culturalmente neutro, basado absolutamente en el desarrollo léxico y gramático-funcional. Los pequeños apartados Did you know...? son los únicos dedicados a algo mínimamente cultural $y$, como indican su nombre mismo, se quedan en hechos anecdóticos. La temática tiende a girar más hacia temas como los animales o la naturaleza. Al igual que NEF, pierde alguna ocasión para profundizar en temas culturalmente relevantes. Particularmente llamativa es la unidad 11B, South is up, en la que se habla de la orientación tradicional de los mapas 
(norte arriba y sur abajo, Europa en el centro), sin entrar a trabajar el etnocentrismo que subyace en este hecho.

Por el contrario, Cambridge ha dedicado mayor atención al aprendizaje cultural con EU. No debemos dejar de mencionar que cada dos unidades encontramos una página (Across cultures) con este fin. Fuera de ellas, y como sucede en NEF y SF, las referencias culturales son británicas. El trabajo realizado para intricar la enseñanza cultural en el aprendizaje de idiomas es obvio y encomiable por parte de esta editorial. Particularmente acertada nos resulta la unidad sobre incidentes críticos y malentendidos interculturales (p.64-65), que supone un magnífico ejemplo de lo que se puede ofertar dentro de materiales curriculares culturalmente conscientes.

El papel de los libros de texto en este sentido ha sido evaluado de manera desigual en la literatura existente. Mientras Cortazzo y Jin (1999) afirman que los libros de texto son portadores de valores culturales y una ventana a la cultura de la segunda lengua, Paige (1999) indica que los textos presentan poca o ninguna explicación acerca de cómo los patrones de comportamiento se desarrollan y se relacionan en el complejo sistema cultural.

Hemos de reseñar la labor más reciente de Varón (2009) y Lee (2009). Aunque el estudio de Varón se centra en la enseñanza del español como lengua extranjera y Lee ha analizado libros coreanos de conversación en lengua inglesa, sus hallazgos son comparables a los que hemos presentado en este estudio. Ambos autores insisten en que la enseñanza cultural ha de estar más sistematizada y ha de permitir un uso más reflexivo de los materiales, con una mayor incidencia en los valores y creencias. Nuestro estudio no hace sino apoyar estas afirmaciones, soportándose en un mayor nivel de concreción y demostrando mediante el análisis por destrezas este hecho.

Entendemos que el fin último ha de ser el de capacitar para aprender, más que el de enseñar una cultura particular. En palabras de Rajagopalan (2004), el inglés es una lengua intercultural. El desarrollo de la $\mathrm{CCl}$ dentro del aula supone una ventaja enorme en contraposición a los encuentros interculturales "reales": se reduce el nivel de estrés que cualquier comunicación de este tipo produce de manera inevitable (Vilà, 2005).

\section{Conclusiones}

El aprendizaje de idiomas en la actualidad ha de estar ligado necesariamente al aprendizaje cultural. Vivimos en una sociedad multicultural que requiere 
desarrollar una competencia comunicativa intercultural que nos capacite plenamente. La corrección formal es insuficiente una vez asumimos la complejidad del concepto lengua y cultura como binomio inseparable. Lejos quedan los años en los que parecía suficiente el aprendizaje mimético del hablante nativo (Kramsch, 2009). La idea de un hablante monolingüe, monocultural, adscrito a una nación concreta, sin influencias de ningún tipo es, cuando menos, una fantasía no aplicable a la realidad multicultural del siglo veintiuno. Por lo tanto, no defendemos la adquisición de conocimientos culturales de un grupo determinado, sino la capacitación cultural del alumnado. La mera transmisión de conocimientos resulta particularmente estéril, máxime en el caso del inglés por su posición como lengua global (Alptekin, 2002).

Además, este tipo de enseñanza puede producir un refuerzo de los estereotipos y una descodificación de los mensajes culturales en base a las propias creencias. Resulta imprescindible desarrollar destrezas y actitudes que hagan competente a nuestro alumnado. Esto no se puede llevar a cabo sin actividades que impliquen la reflexión acerca de los valores y las creencias, propias y ajenas, que subyacen en los comportamientos. Se hace necesario introducir actividades de contraste, de análisis de autoestereotipos y de relativización continua de las generalidades con el fin de que el aprendizaje no quede en lo superficial (Areizaga, 2002; Göbel y Helmke, 2010).

Dentro de nuestro propio análisis, hemos podido observar que existe un estatismo en la creación de materiales por parte de las editoriales. Este se refleja en el hecho de que dentro de la "Cultura en general" se trabaje mayoritariamente la transmisión de información.

Sería injusto no mencionar el esfuerzo de Cambridge, con English Unlimited, que cuadriplica el número de ocasiones en el que se trabaja el aprendizaje cultural en sus textos, haciéndose esta diferencia mucho más patente en el caso de los comportamientos y las actitudes, que son ignoradas por Macmillan y Oxford en sus textos.

Resulta más común ver en un libro una foto del skyline de Manhattan que un ejercicio que permita a los alumnos descubrir qué valores norteamericanos subyacen tras estos (la megalomanía o la obsesión con la frontera, por poner algún ejemplo). El primer tipo de aprendizaje es anecdótico y "turístico", por así llamarlo. El segundo, nos da la llave para comprender en profundidad el primero y ser capaces de interpretar comportamientos nuevos; nos hace conocedores de la cultura en su sentido más amplio y complejo. Se puede 
observar que la mayoría de las menciones culturales son referencias a hechos más que a los valores o creencias que subyacen bajo estos. No son materiales, por tanto, que se ajusten a las necesidades formativas de nuestro alumnado.

Como indica Varón (2009, p.120), "lo que se necesita es la inserción de métodos apropiados para enseñar y aprender la cultura, que facilite un uso reflexivo de los materiales". Sólo de esta manera se podrán desarrollar en el alumnado las competencias requeridas para su necesaria capacitación cultural. Hemos podido observar que después de dos décadas desde la publicación del MCERL, aún no existe una adaptación de los libros de texto con los objetivos expresados en el mismo, aunque estos tampoco fueran exhaustivos en lo que al aprendizaje cultural se refiere. Esta enseñanza se atiende de manera unidireccional e insuficiente en la mayoría de los casos. Por otra parte, hemos podido demostrar el asimétrico uso de las destrezas productivas y receptivas, lo que supone un hallazgo importante en referencia a la implicación del alumnado en este tipo de enseñanza. Sin un mayor uso de las destrezas productivas, no podemos asumir una involucración suficiente del alumnado. Una enseñanza basada en la transmisión de información conceptual no puede desarrollar actitudes y habilidades ni puede promover un aprendizaje significativo.

\section{About the author}

Antonio Raigón es Profesor Ayudante Doctor del Departamento de Filologías Inglesa y Alemana de la Universidad de Córdoba, España. Sus líneas de investigación son la formación intercultural del profesor de lengua extranjera, la comunicación intercultural y ha realizado estudios acerca de los procedimientos de acreditación de competencia lingüística. Publicaciones: Raigón, A. y Gómez, E. (2005). The influence of ICTS in vocabulary learning: An empirical study. Porta Linguarum (4) 87-107; Gómez, E. y Raigón, A. (2009). The intercultural training of foreign language teachers: The Spanish case. El Guiniguada (18) 53-66; Larrea, A., Raigón, A. y Gómez, E. (2012). ICT for intercultural competence development. Pixel-Bit (40) 115-124; Raigón, A. y Larrea, A. (2013). Análisis y percepción por parte del alumnado del requisito lingüístico en el acceso al Máster de Formación del Profesorado de Educación Secundaria. Porta Linguarum (20) 63-76; Raigón, A. (2015). The impact of introducing foreign language 
requirements on the Spanish higher education system: The case of Córdoba University. Language Learning in Higher Education (5.1) 265-279.

\section{LFE Article history}

Paper received: 18th November 2015

Paper received in revised form and accepted for publication: 19th May 2015

\section{Referencias}

Aleptkin, C. (2002). Towards Intercultural Communicative Competence in ELT. ELT Journal, 56 (1), 57-64.

Areizaga, E. (2002). El componente cultural en la enseñanza de lenguas: elementos para el análisis y la evaluación del material didáctico. Cultura y Educación, 14 (2), 161-175.

Bennett, J., Bennett, M., y Allen, W. (2003). Developing intercultural competence in the language classroom. En L. Dale, R. Lange y M. Paige (Eds.) Culture as the core: Perspectives in second language learning. Greenwich, CT: Information Age.

Byram, M. (1989).Cultural Studies in Foreign Language Education. Clevedon, U.K.: Multilingual Matters.

Byram, M. (1997). Teaching and assessing intercultural communicative competence. Clevedon: Multilingual Matters.

Byram, M. (2000). Assessing intercultural competencein language teaching. Sprogforum, 18, 8-13.

Byram, M. (ed.) (2001). Languages for Intercultural Communication and Education, 1: Developing Intercultural Competence in Practice. Clevendon: Multilingual Matters Limited.

Consejo de Europa (2002). Marco de Referencia Europeo para el Aprendizaje, la Enseñanza y la Evaluación de Lenguas. Madrid: Instituto Cervantes.

Cortazzi, M. y Jin L. (1999). Cultural Mirrors, Materials and Methods in the EFL Classroom. En E. Hinkel (Ed.), Culture in Second Language Teaching and Learning. Cambridge: Cambridge University Press.

Council of Europe (2001). Common European Framework of Reference for Languages: Learning, Teaching, Assessment. Cambridge: Cambridge University Press.

Deardorff, K. (2006). Identification and assessment of intercultural competence as a student outcome of internationalization. Journal of Studies in International Education, 10, 241-266. 
Genc, B. y E. Bada (2005). Culture in Language Learning and Teaching.The Reading Matrix, 5(1), 73-84.

Göbel, K. y Helmke, A. (2010). Intercultural learning in English as foreign language instruction: The importance of teachers' intercultural experience and the usefulness of precise instructional directives. Teaching and Teacher Education, 26, 1571-1582.

Hendson, U. S. (2005). The interaction of language and culture: new views in foreign language teaching. In W. Gehring (Ed.), Kulturdidaktikim Englischunterricht. Oldenburg: Universität Oldenburg.

Hofstede, G. (2001). Culture's consequences: Comparing values, behaviours, institutions and organizations across nations. Thousand Oaks, CA: Sage Publication.

Holliday, A., Hyde, M., y Kulman, J. (2004).Intercultural Communication: An advanced Resource Book. London: Routledge.

Hyde, M. (1998).Intercultural Competence in English Language Education. Modern English Teacher, 7(2), 7-11.

Kramsch, C. (1993). Context and Culture in Language Teaching. Oxford: Oxford University Press.

Kramsch, C. (1998). El privilegio del hablante intercultural. En M. Byram y M. Fleming (Eds.) Perspectivas Interculturales en el Aprendizaje de Idiomas. Cambridge: C.U.P.

Lakoff, G. (1987). Women, Fire and Dangerous Things. What Categories Reveal about the Mind. Chicago: University of Chicago Press.

Larzen-Osternmark, E. (2009). Language teacher education in Finland and the cultural dimension of foreign language teaching - a student teacher perspective. European Journal of Teacher Education, 32, 401-21.

Lee, K. (2009). Treating culture: What 11 high school EFL conversation textbooks in South Korea do. English Teaching: Practice and Critique, 8 (1), 76-96.

Lee, K. (2012). Teaching intercultural English learning/teaching in world Englishes. English Teaching: Practice and Critique, 11(4), 190-205.

Liaw, M. (2006). E-learning and development of intercultural competence. Language Learning and Technology, 10 (3), 49-64.

Mitchell, R., \& Myles, F. (2004).Second language learning theories. London: Arnold.

Novinger, T. (2001). Intercultural Communication: A Practical Guide. Austin: University of Texas Press.

Paige, R., Jorstad, J., Paulson, L., Klein, F., y Colby, J. (1999). Culture learning in language education: A review of the literature. En R. Paige, D. Lange, y Y.Yershova (Eds.), Culture as the core: Integrating culture into the language curriculum. Minneapolis, MN: The Center for Advanced Research on Language Acquisition, University of Minnesota.

Paricio, María S. (2005). La dimensión cultural en los libros de texto de lenguas extranjeras; pautas para su análisis. Glosas didácticas, 15, 133-144. 
Pusch, M. y La Brack, B. (2003). Professional Practice Workshop: Fundamentals of Intercultural Communication. Portland: Association of International Educators, Professional Development Program.

Rajagopalan, K. (2004). The concept of world Englishes and its implication for ELT. ELT Journal, 58(2), 111-117.

Risager, K. (1998). Language teaching and the process of European integration. En M. Byram, y M. Fleming (Eds.), Language Learning in Intercultural Perspective. Cambridge: Cambridge University Press.

Rodrigo, M. (1999). La comunicación intercultural. Barcelona: Anthropos.

Sapir, E. (1958). Culture, Language and Personality. Berkeley, CA: University of California Press.

Scheu, D. (2005). Linguistic and cultural relativity in foreign language education. En W. Gehring (Ed.), Kulturdidaktikim Englischunterricht. Oldenburg: Universität Oldenburg.

Sercu, L. (2000). Textbooks. En Routledge Enciclopedia of Language Teaching and Learning, Byram, M. (ed.). Londres: Routledge.

Sercu, L. (2005). Teaching foreign languages in an intercultural world. En M. Byram y A. Phipps (Eds.), Foreign language teachers and intercultural competence. Buffalo, NY: Multilingual Matters, Ltd.

Varón Páez, M. (2009).Componente cultural, libros de texto y enseñanza del inglés como lengua extranjera. Forma y Función, 22 (1), 95-124.

Vez, J. M. (2001). Formación en didáctica de las lenguas extranjeras. Rosario, Argentina: Homo Sapiens.

Vilà Baños, R. (2004). Una actividad telemática para el desarrollo de la competencia comunicativa intercultural de adolescentes. En E. Soriano (Coord.), Recursos para la Enseñanza y Aprendizaje de la Educación Intercultural. Almería: Universidad de Almería.

Vilà Baños, R. (2005). La competencia comunicativa intercultural. Un estudio en el Primer Ciclo de la ESO. Tesis Doctoral. Barcelona: Universitat de Barcelona.

Ware, P., \& Kramsch, C. (2005). Toward an intercultural stance: Teaching German and English through telecollaboration. The Modern Language Journal, 89(2), 190-205.

Weaver, G. (1986). Understanding and coping with cross-cultural adjustment stress. En R. M. Paige (Ed.),Cross-Cultural Orientation: New Conceptualizations and Applications. Lanham, MD: University Press of America.

Widdowson, H. G. (1998). Context, Community and Authentic Language. TESOL Quarterly, 32(4), 705-716. 